\title{
Emotional reactivity and interoceptive sensitivity: Exploring the role of age
}

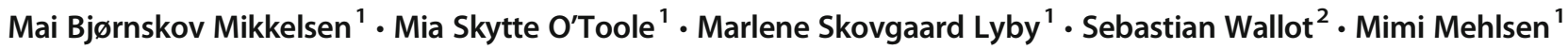

Published online: 25 April 2019

(C) The Psychonomic Society, Inc. 2019

\begin{abstract}
Interoceptive sensitivity (IS) refers to the ability to accurately perceive visceral afferent information, and several prominent theories of emotions suggest that IS is associated with heightened emotional reactivity. Recent evidence has pointed to a decline in IS with age, but there is no consistent evidence of age-related decline in emotional reactivity. This may be because the relationship between IS and emotional reactivity changes with age. To address this hypothesis, we examined the moderating role of age in the association between IS and emotional responses to affect-inducing images. A sample of 65 young adults (mean age $=23.91$ years, $S D=4.62$ ) and 32 older adults (mean age $=61.78$ years, $S D=8.76$ ) was exposed to affect-inducing images from the Nencki Affective Picture System database and completed a heartbeat perception task. Participants' subjective emotional responses to the images were assessed with questionnaires, and their physiological reactivity was indicated by electrodermal activity, heart rate, and heart rate variability during image viewing. The results revealed that age moderated the association between IS and emotional reactivity, while no significant age differences were found in IS, change in affect, or physiological reactivity. The findings demonstrated that IS was associated with emotional reactivity for young adults but not for older adults, suggesting that young and older adults may differ in their use of internal bodily signals to obtain information about their emotional experience. Consistent with contemporary developments within the affective sciences, the results emphasize the importance of individual differences in emotional experiences.
\end{abstract}

Keywords Interoception $\cdot$ Interoceptive sensitivity $\cdot$ Emotion $\cdot$ Emotion processing and aging

Interoception refers to the sensation of the physiological state of the body (Craig, 2002) and has increasingly been a topic of interest within the field of psychology. Introceptive sensitivity (IS) is an integral component of interoception and refers to the ability to accurately perceive visceral afferent information (Garfinkel \& Critchley, 2013). IS has been given credence as a central constituent of emotional experiences in many peripheral theories of emotions (i.e., theories where physiological changes are proposed to underpin emotional experiences;

Electronic supplementary material The online version of this article (https://doi.org/10.3758/s13423-019-01603-y) contains supplementary material, which is available to authorized users.

Mai Bjørnskov Mikkelsen

mai@psy.au.dk

1 Department of Psychology and Behavioral Sciences, Aarhus University, Aarhus, Denmark

2 Max Planck Institute for Empirical Aesthetics, Frankfurt, Germany e.g., Damasio, 1999; James, 1884; Schachter \& Singer, 1962). One of the most well-known and well-cited peripheral theories of emotions originates from William James, who argued that emotional experiences can be reduced to the perception of physiological changes. The importance of physiology for emotions has further been acknowledged in the more recent somatic marker hypothesis by Damasio (1994, 1999). Damasio proposed that neural mapping of visceral changes contributes to emotion generation, but added that the experience of an emotion is inherently shaped by cognition. Although peripheral theorists such as James and Damasio differ in the emphasis they place on the contribution of other psychological constructs to the generation and experience of emotions, they share the contention that the perception and representation of physiological changes provide the foundation for the experience of emotions.

IS is often quantified using heartbeat detection tasks, because heartbeats represent discrete internal events that can be easily measured (Garfinkel, Seth, Barrett, Suzuki, \& Critchley, 2015). Heartbeat detection tasks typically entail 
counting the number of perceived heartbeats within a prespecified time period, and performance is summarized as the difference between the perceived number of heartbeats and the actual number of heartbeats during a series of fixed temporal intervals (Schandry, 1981). Previous research has demonstrated a link between heartbeat perception and the perception of changes in other autonomically innervated organs (Harver, Katkin, \& Bloch, 1993; Herbert, Muth, Pollatos, \& Herbert, 2012; Whitehead \& Drescher, 1980; however, see Pennebaker, Gonder-Frederick, Cox, \& Hoover, 1985). As such, heartbeat perception tasks can be considered valid measures of general sensitivity to the physiological state of the body.

Following the central tenets of peripheral theories of emotions, IS should be associated with the intensity of emotional experiences. A growing body of research has confirmed this association using heartbeat detection tasks. Pollatos and colleagues have repeatedly demonstrated a positive association between IS and emotional reactivity (i.e., subjective change in emotional experience following exposure to triggering stimuli; Pollatos, Gramann, \& Schandry, 2007; Pollatos, Herbert, Matthias, \& Schandry, 2007; Pollatos \& Schandry, 2008). Similar results have been reported by Wiens, Mezzacappa, and Katkin (2000) using affect-inducing film clips. Outside of the lab, Barrett, Quigley, Bliss-Moreau, and Aronson (2004) reported that greater IS was associated with increased focus on arousal during an experience-sampling procedure. Taken together, these lines of evidence indicate that individual differences in IS are related to the intensity of subjectively felt emotional experience.

The results from two studies suggest that cardiac IS declines with age well into late adulthood (Khalsa, Rudrauf, \& Tranel, 2009; Murphy, Geary, Millgate, Catmur, \& Bird, 2018). However, because IS was operationalized in two different ways in these studies, further research will be needed before definitive conclusions can be drawn about the development of cardiac IS in adulthood. Age-related decline has been reported in the perception of other visceral changes, such as gastric distension (Rayner, MacIntosh, Chapman, Morley, \& Horowitz, 2000), pain (Lautenbacher, Peters, Heesen, Scheel, \& Kunz, 2017), and thirst (Kenney \& Chiu, 2001). Complementary to these findings, age-related decline in brain volume is apparent in regions considered central to IS, including somatosensory and insular cortices (Good et al., 2001; Salat et al., 2004). The available empirical evidence thus points to reduced IS with increasing age.

Despite this possible decline in IS across the adult life span, there is no consistent evidence of age-related decline in emotional reactivity (e.g., Carstensen et al., 2011; Mikkelsen, Mehlsen, \& O'Toole, 2018). Accordingly, it would be interesting to investigate whether IS and its association with emotional experiences remain stable across the adult life span.
The association between IS and the intensity of emotional experiences may diminish with age. Indeed, maturational dualism (Mendes, 2010) is the idea that the link between mind and body weakens with age, and that this weakening has important consequences for emotional experiences (Mendes, 2010). According to maturational dualism, a combination of age-related reductions in IS and in physiological reactivity results in impairment of the ability to use internal bodily states as a source of information to guide decision-making and behavior. Consequently, older adults rely more on their external environments and less on their internal bodily states to obtain information about their emotional states than young adults do.

Although the tenets of maturational dualism have yet to be systematically explored, a few studies appear to substantiate the idea. One line of evidence comes from studies of emotionbased learning using the Iowa Gambling Task (IGT; Bechara, Damasio, Tranel, \& Damasio, 1997). The IGT is a decisionmaking task in which players choose cards from one of four decks, winning or losing money with each choice. Performance on the IGT is believed to be contingent on the ability to let information from one's internal bodily state inform decision-making (Bechara, Damasio, \& Damasio, 2000; Bechara, Damasio, Tranel, \& Damasio, 2005). In support of maturational dualism, older adults have been shown to perform worse on the IGT than do young adults (Beitz, Salthouse, \& Davis, 2014; Denburg, Tranel, \& Bechara, 2005; Fein, McGillivray, \& Finn, 2007; but see Wood, Busemeyer, Koling, Cox, \& Davis, 2005). Findings from this sparse body of research appear to support age differences in the use of internal bodily states to obtain information about emotional experiences. Whether these age differences reflect a weakened association between IS and emotional experiences remains to be determined and could potentially have important implications for the understanding of emotional development in adulthood.

The purpose of the present study was to explore whether the association between IS and emotional reactivity depends on age. On the basis of the existing literature, we hypothesized that the association between IS and emotional reactivity would be moderated by age and be stronger for young than for older adults. In other words, awareness of one's internal state was expected to have a larger impact on young adults' judgments of their emotional reactions than on older adults' judgments. Because no previous study of IS and emotional reactivity has included measures of change in general affect (i.e., not directly in response to affect-inducing stimuli), we further investigated age differences in the association between IS and change in positive and negative affect from baseline to after exposure to affect-inducing stimuli. Finally, physiological measures of reactivity were included in order to investigate whether there were age differences in the associations between physiological and emotional reactivity. 


\section{Method}

\section{Participants}

Participants were recruited through advertisement online. A total of 101 Danish-speaking adults with no history of heart conditions provided written informed consent and were enrolled in the study. Four participants were excluded from the analyses because it was not possible to obtain accurate IS scores for them, due to artifacts in their electrocardiogram recordings. Thus, the final sample consisted of 65 young adults (19-46 years old, $M=23.91, S D=4.62 ; 52.3 \%$ female) and 32 older adults (50-77 years old, $M=61.78, S D=8.761$; $46.9 \%$ female). The participants were compensated with a 250 DKK (approximately \$40) gift card.

\section{Materials}

Affect-inducing images were selected from the Nencki Affective Picture System database (Marchewka, Żurawski, Jednoróg, \& Grabowska, 2014). A total of 105 images validated to induce disgust $(n=35)$, sadness $(n=35)$, and mixed negative emotions $(n=35)$ were chosen on the basis of normative data provided by Riegel et al. (2016). Disgust- and sadness-inducing images were chosen because previous research has indicated that disgust and sadness are easier to elicit with visual stimuli than are other emotions (Gross \& Levenson, 1995; Maffei et al., 2014). The images were validated in a sample of 57 young and older Danish adults, who rated the images on scales of valence $(1=$ negative, $9=$ positive $)$ and arousal $(1=$ relaxed, $9=$ aroused $)$. These ratings provided the basis for creating two sets of 50 images with no significant age-group differences in overall mean ratings of valence and overall mean ratings of arousal (see the supplementary material).

\section{Measures}

Self-report Emotional reactivity was assessed by means of a visual analog scale (VAS) ranging from 0 (not at all affected by the images) to 100 (extremely affected by the images). Positive and negative affective states were measured with the positive and negative affect schedule (PANAS; Watson, Clark, \& Tellegen, 1988) using the "right now" instruction.

Physiological measures Continuous recordings were made of electrodermal activity and of the electrical activity of the heart starting $5 \mathrm{~min}$ before stimulus presentation and continuing throughout the experimental procedure. For details on the physiological data recording, see the supplementary material.

\section{Procedure}

Participants were equipped with physiological sensors and seated in front of a 22-in. monitor located approximately 0.5 $\mathrm{m}$ away from them. The experimental procedure started with a 5-min baseline recording, during which participants were instructed to remain as still as possible. Following the baseline recording, participants filled in the PANAS and received the instruction: "You will now be shown a series of images. Look at the images carefully. Do not look away." Subsequently, participants were randomly assigned to view one of the two image sets. Each image was presented for $5 \mathrm{~s}$ with a 1 -s blank interstimulus interval, and the image presentation order was randomized within each set. After viewing the images, participants rated how emotionally affected they were by them and then filled in the PANAS again. There were no differences in IS $[t(95)=0.68, p=.499]$, emotional reactivity $[t(95)=0.71, p$ $=.478]$, change in positive affect $[t(95)=0.27, p=.790]$, or change in negative affect $[t(95)=0.35, p=.726]$ between participants who viewed the two different image sets. Participants were then administered a heartbeat detection task following the guidelines by Schandry (1981). Accordingly, participants silently counted their heartbeats for intervals of $25,35,45$, and $55 \mathrm{~s}$ and reported the number of perceived heartbeats at the end of each interval. The order of the intervals was randomized, and there was no difference in IS between the participants who started with long intervals (45-55 s) and the participants who started with short intervals $(25-35 \mathrm{~s})$ $[t(72.02)=0.25, p=.802]$.

\section{Statistical analysis}

Following Herbert, Herbert, and Pollatos (2011), IS was assessed by calculating an accuracy score for the heartbeat detection task according to Eq. 1:

$\sum(1-\mid$ recorded heartbeats-counted heartbeats $\mid) /$ recorded heartbeats $)$.

The accuracy scores range from 0 to 1 , with higher scores indicating higher accuracy — that is, better IS.

Emotional reactivity was assessed as the raw score on the VAS of how emotionally affected participants felt by the images. Change in positive affect (PA) and change in negative affect (NA) were calculated as residualized gain scores derived from regressions of baseline affect on affect following the image viewing. Residualized gain scores quantify changes in scores after accounting for mean changes in participants and are more reliable than crude change scores (Cronbach \& Furby, 1970). There were no missing data for the self-report measures, since participants were unable to progress in the questionnaires unless they had answered all items. 
Physiological reactivity was assessed during image viewing as (1) average skin conductance level (SCL), (2) heart rate (HR), and (3) heart rate variability (HRV; see the supplementary material for details on the physiological data processing). These measures were selected to provide physiological measures associated with the valence (HRV) and arousal (HR, SCL) of felt emotion (Kreibig, 2010). Due to artifacts in the physiological data, it was not possible to obtain SCL measures from three participants and HR and HRV measures from five participants. Regressions of baseline physiological reactivity on physiological reactivity during the image viewing were conducted in order to obtain residualized gain scores for physiological reactivity.

Independent-samples $t$ tests were used to assess age-group differences in IS, emotional responses, and physiological reactivity. To address the main hypothesis - namely that the association between IS and emotional reactivity would be moderated by age - moderator analyses were conducted using a regression-based macro for SPSS (PROCESS; Hayes, 2012). All continuous predictor variables were standardized prior to the analysis, to minimize multicollinearity. Three moderator analyses were conducted with age as a continuous moderator of the effect of IS on (1) emotional reactivity, (2) change in PA, and (3) change in NA. Previous research has indicated that IS may be linked to variables that may also be influenced by aging, such as body-mass index, physiological reactivity, and self-reported IS accuracy (Knapp-Kline \& Kline, 2005; Pollatos, Herbert, Kaufmann, Auer, \& Schandry, 2007; Ring, Brener, Knapp, \& Mailloux, 2015). To avoid partialing fallacy problems (i.e., using a covariate that is a defining part of the phenomena of interest; cf. Jaccard, Guilamo-Ramos, Johansson, \& Bouris, 2006), no attempt was made to control for these variables in the main analyses. Post-hoc analyses were conducted in order to detail the findings from the moderator analyses.

\section{Results}

\section{Age-group differences in interoceptive sensitivity, emotional reactivity, change in affect, and physiological reactivity}

Table 1 shows IS performance, mean ratings of emotional reactivity, change in affect, and change in physiological reactivity for the two age groups, as well as results from analyses of age-group differences.

As is evident from Table 1, there were no age-group differences in IS, emotional reactivity, changes in affect, baselinecorrected HR, or baseline-corrected SCL. However, a significant age-group difference in HRV was observed: Older adults experienced a significantly greater increase in HRV from baseline to image viewing, as compared to young adults. For further analyses of age-group differences, see the supplementary material.

\section{Moderating role of age}

Moderator analyses were performed to examine whether age affected the relationships between IS and emotional reactivity and between IS and changes in affect.

Age as a moderator of the relationship between IS and emotional reactivity Age was investigated as a potential moderator of the effect of IS on emotional reactivity. The results revealed that IS had a significant independent direct effect on emotional reactivity, while age did not (see Table 2, Model 1). The interaction term, IS $\times$ age, was significant, explaining a significant increase in variance in emotional reactivity $\left[\Delta R^{2}=.06\right.$; $\left.F(1,93)=6.61, p=.012, f^{2}=.16\right]$. Thus, age was a moderator of the relationship between IS and emotional reactivity.

Table 1 Interoceptive sensitivity, mean ratings of emotional reactivity, changes in affect, and changes in physiological reactivity for the two age groups, along with results from analyses of age-group differences

\begin{tabular}{|c|c|c|c|c|}
\hline & Young adults & Older adults & $\begin{array}{l}\text { Age-group differences } \\
t(d f)\end{array}$ & $\begin{array}{l}\text { Effect size } \\
d\end{array}$ \\
\hline Interoceptive sensitivity, Mean $(S D)$ & $0.64(0.23)$ & $0.68(0.22)$ & $1.00(95)^{\text {n.s. }}$ & 0.18 \\
\hline Emotional reactivity, Mean $(S D)$ & $49.51(25.34)$ & $56.66(26.45)$ & $1.29(95)^{\text {n.s. }}$ & 0.28 \\
\hline Change in NA, Mean $(S D)$ & $4.00(3.84)$ & $5.31(4.92)$ & $1.38(95)^{\text {n.s. }}$ & 0.30 \\
\hline Change in PA, Mean $(S D)$ & $-3.45(3.38)$ & $-4.25(4.79)$ & $0.89(95)^{\text {n.s. }}$ & 0.19 \\
\hline HR, Mean $(S D)$ & $3.51(5.49)$ & $3.05(3.15)$ & $0.40(90)^{\text {n.s. }}$ & 0.10 \\
\hline HRV, Mean $(S D)$ & $0.12(0.64)$ & $0.38(0.62)$ & $2.82(38.35)^{*}$ & 0.41 \\
\hline SCL, Mean $(S D)$ & $0.82(1.81)$ & $0.62(2.07)$ & $1.47(92)^{\text {n.s. }}$ & 0.14 \\
\hline
\end{tabular}

n.s. Nonsignificant-i.e., $p>.05 .{ }^{*}$ Significant at $p<.05$. Note that while the means and $S D$ s for positive affect (PA) and negative affect (NA) reflect changes in raw scores, and the means and $S D$ s for the physiological measures reflect changes from baseline to image viewing, the $t$ tests were performed with residualized gain scores. A Levene's test for equality of variances indicated that equality could not be assumed for heart rate variability $(F=5.75, p=$ .019), so the degrees of freedom were adjusted for this measure. HR, heart rate; HRV, heart rate variability; SCL, skin conductance level 
Table 2 Results from regression analyses showing the moderating effect of age on the associations between interoceptive sensitivity (IS) and emotional reactivity (Model 1), between IS and change in negative affect (NA; Model 2) and between IS and change in positive affect (PA; Model 3)

\begin{tabular}{|c|c|c|c|c|c|c|c|c|c|}
\hline & \multicolumn{3}{|c|}{$\begin{array}{l}\text { Model 1: } \\
\text { IS predicting emotional reactivity }\end{array}$} & \multicolumn{3}{|c|}{$\begin{array}{l}\text { Model 2: } \\
\text { IS predicting change in NA }\end{array}$} & \multicolumn{3}{|c|}{$\begin{array}{l}\text { Model 3: } \\
\text { IS predicting change in PA }\end{array}$} \\
\hline & $B(S E)$ & $p$ & $95 \% \mathrm{CI}$ & $B(S E)$ & $p$ & $95 \% \mathrm{CI}$ & $B(S E)$ & $p$ & $95 \% \mathrm{CI}$ \\
\hline \multicolumn{10}{|c|}{ Direct effects } \\
\hline IS & $-24.17(11.05)$ & .031 & $-46.12 ;-2.22$ & $-4.21(1.84)$ & .024 & $-7.86 ;-0.56$ & $1.96(1.61)$ & .226 & $-1.24 ; 5.16$ \\
\hline Age & $0.17(0.13)$ & .197 & $-0.09 ; 0.44$ & $0.03(0.02)$ & .139 & $-0.01 ; 0.08$ & $0.02(0.02)$ & .320 & $-0.02 ; 0.06$ \\
\hline \multicolumn{10}{|l|}{ Interaction } \\
\hline IS $\times$ Age & $1.50(0.58)$ & .012 & $0.34 ; 2.66$ & $0.17(0.10)$ & .088 & $-0.03 ; 0.36$ & $-0.01(0.09)$ & .924 & $-0.18 ; 0.16$ \\
\hline
\end{tabular}

Age as a moderator of the relationship between IS and change in NA When we examined age as a potential moderator of the relationship between IS and change in NA, the results revealed that IS exerted a significant independent direct effect on NA, while age did not (see Table 2, Model 2). In addition, age failed to demonstrate a moderating effect on the association between IS and NA $\left[\Delta R^{2}=.03 ; F(1,93)=2.97, p=.088\right.$, $\left.f^{2}=.07\right]$.

Age as a moderator of the relationship between IS and change in PA Moderator analyses were conducted with age entered as a potential moderator of the effect of IS on change in PA. The results revealed that neither IS nor age had a direct effect on change in PA (see Table 2, Model 3). In addition, age was not found to be a moderator of the association between IS and PA $\left[\Delta R^{2}=<.01 ; F(1,93)=0.19, p=.924, f^{2}<.01\right]$.

\section{Post-hoc analyses of effects of age}

Since the main analyses indicated that age moderated the effect of IS on emotional reactivity, post-hoc analyses were performed to explore this finding. Pearson's correlation analyses indicated a significant inverse relationship between IS and emotional reactivity for young adults $(r=-$ $.38, p=.002)$, while no significant relationship was apparent for older adults $(r=.11, p=.554)$. The age groups were split into subgroups based on median IS scores, resulting in four groups: 32 young individuals performing well (i.e., above the median for their age group) and 33 young individuals performing poorly (i.e., below the median for their age group), as well as 16 older individuals performing well and 16 older individuals performing poorly. Because the data were normally distributed for both age groups, independent $t$ tests were applied in order to compare emotional reactivity between the individuals performing well and the individuals performing poorly within each age group. For the young adults, individuals who performed well reported significantly lower levels of emotional reactivity $(M=$ 43.22, $S D=24.26$ ) as compared with individuals who performed poorly $(M=55.61, S D=25.22)[t(63)=2.02$, $p=.048]$. No significant differences were found for the older adults, with individuals who performed well $(M=$ $58.31, S D=26.56)$ and individuals who performed poorly $(M=55.00, S D=27.09)$ reporting similar levels of emotional reactivity $[t(30)=0.35, p=.729]$.

Age-group differences in the association between emotional reactivity and physiological reactivity Because IS was measured as a general ability, it does not provide direct evidence of age-group differences in IS during image viewing. Therefore, an analysis was undertaken to explore whether the association between physiological and subjective responses differed between the two age groups, since this could be an indicator of age differences in emotionprocessing systems.

To test age-group differences in the association between emotional reactivity and physiological reactivity, correlation coefficients between emotional reactivity and physiological reactivity were converted into $z$ scores using Fisher's $r$-to-z transformation, and the resulting $z$ scores were compared using Formula 2.8.5 from Cohen and Cohen (1983). The results indicated a significant difference in the correlation between emotional reactivity and residualized gain scores for SCL (young $r=.21$, older $r$ $=-.26 ; z=2.1, p=.047)$, and no significant difference in the correlation between emotional reactivity and residualized gain scores for HR (young $r=.07$, older $r=$ $.35 ; z=1.23, p=.219$ ) and HRV (young $r=-.08$, older $r=$ $-.39 ; z=1.38, p=.168)$.

\section{Discussion}

Despite a growing body of literature on interoceptive sensitivity and emotional experiences, very little research has investigated whether aging influences the association between these constructs. In the present study we addressed this gap in the literature and demonstrated that the 
association between IS and emotional reactivity to negative affect-inducing images was moderated by age. In support of our hypothesis, young adults showed a stronger association between IS and emotional reactivity than did older adults. Post-hoc analyses indicated that young individuals who performed well on the IS task reported significantly lower emotional reactivity than did young individuals who performed poorly, while there was no difference in emotional reactivity between the older individuals who performed well and the older individuals who performed poorly. In addition, the association between physiological reactivity and emotional reactivity differed for the two age groups; however, these results do not lend themselves to a clear-cut interpretation, since they are based on residualized gain scores of the physiological variables.

Taken together, the results indicate that young adults' emotional reaction was associated with their IS while older adults' emotional reaction was not associated with their IS, possibly implying that young and older adults differed in their sensitivity to their internal bodily states when reacting emotionally to the images. The findings represent an important contribution to research on lifespan development in emotions, because they indicate that subjective emotional reactions, physiological reactions, and IS remain stable across the adult life span, while the functional relationship between these variables changes. Regarding the results relating to affect, we found no moderating effect of age on the association between IS and changes in affect measured by the PANAS. This may in part be because participants were asked to report on their global affective state (i.e., not in relation to the images), possibly reducing the sensitivity of the instrument to emotional reactions to the images. The results may thus imply that age differences are less pronounced when adults report on their affective state at a more general level, as opposed to specifically related to an affect-inducing stimulus.

Theoretical work on emotional development in adulthood may shed light on the age differences found in the present study. One possible mechanism underlying the findings may be age-related shifts in emotion processing due to declines in cognitive resources. According to the dynamic integration theory (DIT; Labouvie-Vief \& Medler, 2002), an important distinction can be drawn between optimization (i.e., quick, schematic processing based on existent knowledge structures and aimed at maintaining an optimal affective balance) and differentiation (i.e., elaborate, systematic processing focusing on differentiation of existing knowledge structures) as two central modes of processing information. According to DIT, agerelated cognitive decline makes it increasingly difficult to engage in differentiated processing, and older adults may therefore come to rely on optimization. The age differences in the present study, then, may indicate that young and older adults used different modes of processing when reporting on their reactions to the images. Young adults may have engaged in elaborate processing of their internal emotional states, while older adults may in a more rigid manner have applied schematic knowledge of emotional reactions (i.e., experience-based knowledge of the typical response patterns when faced with negative affect-inducing stimuli), as a part of an optimization strategy.

Another possible mechanism underlying the findings lies within the socioemotional selectivity theory (Carstensen, Isaacowitz, \& Charles, 1999). The assumption of this theory is that as time horizons shrink, motivation shifts from a focus on expansive goals (i.e., acquiring knowledge) to emotion goals (i.e., securing balanced emotional states). This motivational shift is accompanied by changes in emotion regulation strategies. Older adults tend to use disengagement strategies (e.g., distancing or exclusively focusing on the positive aspects of a negative situation), whereas young adults favor engagement strategies that promote learning (e.g., reappraisal or reflection on emotions; Scheibe, Sheppes, \& Staudinger, 2015). The findings of the present study may thus be driven by older adults' attempts to disengage from their experience of the images and younger adults' attempts to engage.

A third plausible explanation for the age differences could be developmental changes in the structure and function of the brain. Evidence from brain-imaging studies has indicated reduced volume in the brain areas associated with IS (Good et al., 2001; Salat et al., 2004) and changes in the functional networks involved in emotion processing with increasing age (Castle et al., 2012; Ziaei, Salami, \& Persson, 2017). Together, these changes may contribute to increased dissociation between IS, emotional experiences, and physiological reactions with age. However, the current state of evidence does not permit any clear conclusions about how developmental changes in the brain may relate to IS, emotional experiences, and physiological reactions.

Further research will be necessary to determine the mechanisms behind the present results. The association between IS and emotional reactivity was found to be modest at best, and the results indicated significant individual differences in the importance of IS for judgments of emotional reactivity. Consequently, internal somatovisceral cues may contribute moderately to the emotional experience for some individuals and contribute less for others. In line with contemporary developments within the affective sciences (e.g., Barrett \& Russell, 2014; Kuppens et al., 2017), the results of the present study emphasize the importance of moving from nomothetic theories of emotional experiences to theories that accommodate significant variation at the idiographic level (i.e., at the level of the individual). Such theories will provide a useful framework 
from which researchers can work toward understanding individual differences in emotional experiences and how they impact other phenomena.

\section{Limitations}

The present study has a number of limitations. First, the causal direction between IS and emotional reactivity cannot be established. Second, the heartbeat detection task was completed after participants were exposed to the affect-inducing stimuli. It is possible that participants who experienced intense emotional reactions to the images may have performed better or worse at the heartbeat detection task because they were already focused on their internal state. However, the reverse order would have been problematic, as well, since previous research has indicated that a focus on interoceptive cues may change subsequent perceptions of emotional stimuli (e.g., Pollatos, Laubrock, \& Wittmann, 2014). Third, only three measures of physiological reactivity were included in the study, and it is therefore unclear whether the present results would generalize to other measures of physiological reactivity. Fourth, participants were unable to progress in the questionnaire unless they had answered all items. This design secured complete data, but it may have reduced the credibility of responses, because participants may have been forced to answer questions they did not understand or were not able to provide an answer for.

\section{Conclusion}

To our knowledge, this has been the first study to examine age differences in the association between interoceptive sensitivity and emotional experiences. The results provide evidence of age-related decline in the strength of the relationship between interoceptive sensitivity and emotional reactivity to affect-inducing images. The findings suggest that in contrast to young adults, older adults' emotional reactivity did not depend on interoceptive sensitivity, possibly implying that young and older adults differed in sensitivity to their internal bodily states when determining their emotional reactions. Further research will be needed to elucidate the mechanisms behind the findings and their implications for theoretical accounts of emotional development in adulthood.

Author note None of the data or materials for the experiments reported here are available, and the experiment was not preregistered.

\section{Compliance with ethical standards}

Conflict of interest The authors declare that they have no conflicts of interest.

\section{References}

Barrett, L. F., Quigley, K. S., Bliss-Moreau, E., \& Aronson, K. R. (2004). Interoceptive sensitivity and self-reports of emotional experience. Journal of Personality and Social Psychology, 87, 684. https://doi. org/10.1037/0022-3514.87.5.684

Barrett, L. F., \& Russell, J. A. (Eds.). (2014). The psychological construction of emotion. New York, NY: Guilford Press.

Bechara, A., Damasio, H., \& Damasio, A. R. (2000). Emotion, decision making and the orbitofrontal cortex. Cerebral Cortex, 10, 295-307. https://doi.org/10.1093/cercor/10.3.295

Bechara, A., Damasio, H., Tranel, D., \& Damasio, A. R. (1997). Deciding advantageously before knowing the advantageous strategy. Science, 275, 1293-1295. https://doi.org/10.1126/science.275.5304.1293

Bechara, A., Damasio, H., Tranel, D., \& Damasio, A. R. (2005). The Iowa Gambling Task and the somatic marker hypothesis: Some questions and answers. Trends in Cognitive Sciences, 9, 159-162. https://doi.org/10.1016/j.tics.2005.02.002

Beitz, K. M., Salthouse, T. A., \& Davis, H. P. (2014). Performance on the Iowa Gambling Task: From 5 to 89 years of age. Journal of Experimental Psychology: General, 143, 1677. https://doi.org/10. 1037/a0035823

Carstensen, L. L., Isaacowitz, D. M., \& Charles, S. T. (1999). Taking time seriously: A theory of socioemotional selectivity. American Psychologist, 54, 165-181. https://doi.org/10.1037/0003-066X.54. 3.165

Carstensen, L. L., Turan, B., Scheibe, S., Ram, N., Ersner-Hershfield, H., Samanez-Larkin, G. R., . . . Nesselroade, J. R. (2011). Emotional experience improves with age: Evidence based on over 10 years of experience sampling. Psychology and Aging, 26, 21-33. https://doi. org/10.1037/a0021285

Castle, E., Eisenberger, N. I., Seeman, T. E., Moons, W. G., Boggero, I. A., Grinblatt, M. S., \& Taylor, S. E. (2012). Neural and behavioral bases of age differences in perceptions of trust. Proceedings of the National Academy of Sciences, 109, 20848-20852. https://doi.org/ 10.1073/pnas.1218518109

Cohen, J., \& Cohen, P. (1983). Applied multiple regression/correlation analysis for the behavioral sciences. Hillsdale, NJ: Erlbaum.

Craig, A. D. (2002). How do you feel? Interoception: The sense of the physiological condition of the body. Nature Reviews Neuroscience, 3, 655-666. https://doi.org/10.1038/nrn894.

Cronbach, L. J., \& Furby, L. (1970). How we should measure "change": Or should we? Psychological Bulletin, 74, 68. https://doi.org/10. 1037/h0029382

Damasio, A. (1994). Descartes' error: Emotion, reason, and the human brain. New York, NY: Quill.

Damasio, A. (1999). The feeling of what happens: Body, emotion and the making of consciousness. London, UK: Vintage.

Denburg, N. L., Tranel, D., \& Bechara, A. (2005). The ability to decide advantageously declines prematurely in some normal older persons. Neuropsychologia, 43, 1099-1106. https://doi.org/10.1016/j. neuropsychologia.2004.09.012

Fein, G., McGillivray, S., \& Finn, P. (2007). Older adults make less advantageous decisions than younger adults: Cognitive and psychological correlates. Journal of the International Neuropsychological Society, 13, 480-489. https://doi.org/10.1017/S135561770707052X

Garfinkel, S. N., \& Critchley, H. D. (2013). Interoception, emotion and brain: new insights link internal physiology to social behaviour. Commentary on: "Anterior insular cortex mediates bodily sensibility and social anxiety" by Terasawa et al. (2012). Social Cognitive and Affective Neuroscience, 8, 231-234. https://oi.org/10.1093/ scan $/$ nss 140

Garfinkel, S. N., Seth, A. K., Barrett, A. B., Suzuki, K., \& Critchley, H. D. (2015). Knowing your own heart: Distinguishing interoceptive 
accuracy from interoceptive awareness. Biological Psychology, 104, 65-74. https://doi.org/10.1016/j.biopsycho.2014.11.004

Good, C. D., Johnsrude, I. S., Ashburner, J., Henson, R. N. A., Friston, K. J., \& Frackowiak, R. S. J. (2001). A voxel-based morphometric study of ageing in 465 normal adult human brains. NeuroImage, 14, 21-36. https://doi.org/10.1006/nimg.2001.0786

Gross, J. J., \& Levenson, R. W. (1995). Emotion elicitation using films. Cognition \& Emotion, 9, 87-108. https://doi.org/10.1080/ 02699939508408966

Harver, A., Katkin, E. S., \& Bloch, E. (1993). Signal-detection outcomes on heartbeat and respiratory resistance detection tasks in male and female subjects. Psychophysiology, 30, 223-230. https://doi.org/10. 1111/j.1469-8986.1993.tb03347.x

Hayes, A. F. (2012). PROCESS: A versatile computational tool for observed variable mediation, moderation, and conditional process modeling (White paper). Ohio State University, Columbus, $\mathrm{OH}$. Retrieved from www.afhayes.com/public/process2012.pdf

Herbert, B. M., Herbert, C., \& Pollatos, O. (2011). On the relationship between interoceptive awareness and alexithymia: Is interoceptive awareness related to emotional awareness? Journal of Personality, 79, 1149-1175. https://doi.org/10.1111/j.1467-6494.2011.00717.x

Herbert, B. M., Muth, E. R., Pollatos, O., \& Herbert, C. (2012). Interoception across modalities: On the relationship between cardiac awareness and the sensitivity for gastric functions. PLOS ONE, 7, e36646. https://doi.org/10.1371/journal.pone.0036646

Jaccard, J., Guilamo-Ramos, V., Johansson, M., \& Bouris, A. (2006). Multiple regression analyses in clinical child and adolescent psychology. Journal of Clinical Child and Adolescent Psychology, 35, 456-479. https://doi.org/10.1207/s15374424jccp3503_11

James, W. (1884). What is an emotion? Mind, 9, 188-205. https://doi.org/ 10.1093/mind/os-IX.34.188

Kenney, W. L., \& Chiu, P. (2001). Influence of age on thirst and fluid intake. Medicine \& Science in Sports \& Exercise, 33, 1524-1532. https://doi.org/10.1097/00005768-200109000-00016

Khalsa, S. S., Rudrauf, D., \& Tranel, D. (2009). Interoceptive awareness declines with age. Psychophysiology, 46, 1130-1136. https://doi. org/10.1111/j.1469-8986.2009.00859.x.

Knapp-Kline, K., \& Kline, J. P. (2005). Heart rate, heart rate variability, and heartbeat detection with the method of constant stimuli: Slow and steady wins the race. Biological Psychology, 69, 387-396. https://doi.org/10.1016/j.biopsycho.2004.09.002

Kreibig, S. D. (2010). Autonomic nervous system activity in emotion: A review. Biological Psychology, 84, 394-421. https://doi.org/10. 1016/j.biopsycho.2010.03.010

Kuppens, P., Tuerlinckx, F., Yik, M., Koval, P., Coosemans, J., Zeng, K. J., \& Russell, J. A. (2017). The relation between valence and arousal in subjective experience varies with personality and culture. Journal of Personality, 85, 530-542. https://doi.org/10.1111/jopy.12258

Labouvie-Vief, G., \& Medler, M. (2002). Affect optimization and affect complexity: Modes and styles of regulation in adulthood. Psychology and Aging, 17, 571-588. https://doi.org/10.1037/08827974.17.4.571

Lautenbacher, S., Peters, J. H., Heesen, M., Scheel, J., \& Kunz, M. (2017). Age changes in pain perception: A systematic-review and meta-analysis of age effects on pain and tolerance thresholds. Neuroscience \& Biobehavioral Reviews, 75, 104-113. https://doi. org/10.1016/j.neubiorev.2017.01.039

Maffei, C., Roder, E., Cortesan, C., Passera, F., Rossi, M., Segrini, M., .. . Fossati, A. (2014). Kinematic elicitation of basic emotions: A validation study in an Italian sample. PSYCH, 5, 1065-1078. https://doi. org/10.4236/psych.2014.59119

Marchewka, A., Żurawski, Ł., Jednoróg, K., \& Grabowska, A. (2014). The Nencki Affective Picture System (NAPS): Introduction to a novel, standardized, wide-range, high-quality, realistic picture database. Behavior Research Methods, 46, 596-610. https://doi.org/10. 3758/s13428-013-0379-1
Mendes, W. B. (2010). Weakened links between mind and body in older age: The case for maturational dualism in the experience of emotion. Emotion Review, 2, 240-244. https://doi.org/10.1177/ 1754073910364149

Mikkelsen, M. B., Mehlsen, M., \& O’Toole, M. S. (2018). Agedependent reactivity to affective images: Evidence for variation across emotion categories. Experimental Aging Research, 44, 297 310. https://doi.org/10.1080/0361073X.2018.1477360

Murphy, J., Geary, H., Millgate, E., Catmur, C., \& Bird, G. (2018). Direct and indirect effects of age on interoceptive accuracy and awareness across the adult lifespan. Psychonomic Bulletin \& Review, 25, 11931202. https://doi.org/10.3758/s13423-017-1339-z

Pennebaker, J. W, Gonder-Frederick, L., Cox, D. J., \& Hoover, C. W (1985). The perception of general vs. specific visceral activity and the regulation of health-related behavior. In E. S. Katkin \& S. B. Manuck (Eds.), Advances in behavioral medicine (Vol. 1, pp. 165197). Greenwich, CT: JAI Press.

Pollatos, O., Gramann, K., \& Schandry, R. (2007). Neural systems connecting interoceptive awareness and feelings. Human Brain Mapping, 28, 9-18. https://doi.org/10.1002/hbm.20258

Pollatos, O., Herbert, B. M., Kaufmann, C., Auer, D. P., \& Schandry, R. (2007). Interoceptive awareness, anxiety and cardiovascular reactivity to isometric exercise. International Journal of Psychophysiology, 65, 167-173. https://doi.org/10.1016/j.ijpsycho.2007.03.005

Pollatos, O., Herbert, B. M., Matthias, E., \& Schandry, R. (2007). Heart rate response after emotional picture presentation is modulated by interoceptive awareness. International Journal of Psychophysiology, 63, 117-124. https://doi.org/10.1016/j.ijpsycho. 2006.09.003

Pollatos, O., Laubrock, J., \& Wittmann, M. (2014). Interoceptive focus shapes the experience of time. PLOS ONE, 9, e86934. https://doi. org/10.1371/journal.pone.0086934

Pollatos, O., \& Schandry, R. (2008). Emotional processing and emotional memory are modulated by interoceptive awareness. Cognition \& Emotion, 22, 272-287. https://doi.org/10.1080/ 02699930701357535

Rayner, C. K., MacIntosh, C. G., Chapman, I. M., Morley, J. E., \& Horowitz, M. (2000). Effects of age on proximal gastric motor and sensory function. Scandinavian Journal of Gastroenterology, 35, 1041-1047. https://doi.org/10.1080/003655200451153

Riegel, M., Żurawski, Ł., Wierzba, M., Moslehi, A., Klocek, Ł., Horvat, M., . . Marchewka, A. (2016). Characterization of the Nencki Affective Picture System by discrete emotional categories (NAPS BE). Behavior Research Methods, 48, 600-612. https://doi.org/10. 3758/s13428-015-0620-1

Ring, C., Brener, J., Knapp, K., \& Mailloux, J. (2015). Effects of heartbeat feedback on beliefs about heart rate and heartbeat counting: A cautionary tale about interoceptive awareness. Biological Psychology, 104, 193-198. https://doi.org/10.1016/j.biopsycho. 2014.12.010

Salat, D. H., Buckner, R. L., Snyder, A. Z., Greve, D. N., Desikan, R. S., Busa, E., . . . Fischl, B. (2004). Thinning of the cerebral cortex in aging. Cerebral Cortex, 14, 721-730. https://doi.org/10.1093/ cercor/bhh032

Schachter, S., \& Singer, J. (1962). Cognitive, social, and physiological determinants of emotional state. Psychological Review, 69, 379. https://doi.org/10.1037/h0046234.

Schandry, R. (1981). Heart beat perception and emotional experience. Psychophysiology, 18, 483-488. https://doi.org/10.1111/j.14698986.1981.tb02486.x

Scheibe, S., Sheppes, G., \& Staudinger, U. M. (2015). Distract or reappraise? Age-related differences in emotion-regulation choice. Emotion, 15, 677. https://doi.org/10.1037/a0039246

Watson, D., Clark, L. A., \& Tellegen, A. (1988). Development and validation of brief measures of positive and negative affect: The 
PANAS scales. Journal of Personality and Social Psychology, 54, 1063. https://doi.org/10.1037/0022-3514.54.6.1063

Whitehead, W. E., \& Drescher, V. M. (1980). Perception of gastric contractions and self-control of gastric motility. Psychophysiology, 17, 552-558. https://doi.org/10.1111/j.1469-8986.1980.tb02296.x

Wiens, S., Mezzacappa, E. S., \& Katkin, E. S. (2000). Heartbeat detection and the experience of emotions. Cognition \& Emotion, 14, 417-427. https://doi.org/10.1080/026999300378905

Wood, S., Busemeyer, J., Koling, A., Cox, C. R., \& Davis, H. (2005). Older adults as adaptive decision makers: Evidence from the Iowa Gambling Task. Psychology and Aging, 20, 220-225. https://doi. org/10.1037/0882-7974.20.2.220
Ziaei, M., Salami, A., \& Persson, J. (2017). Age-related alterations in functional connectivity patterns during working memory encoding of emotional items. Neuropsychologia, 94, 1-12. https://doi.org/10. 1016/j.neuropsychologia.2016.11.012

Publisher's note Springer Nature remains neutral with regard to jurisdictional claims in published maps and institutional affiliations. 